\title{
The low-energy scale of the periodic Anderson model
}

\author{
Th. Pruschke ${ }^{a}$, R. Bulla ${ }^{b}$ and M. Jarrell ${ }^{c}$ \\ ${ }^{a}$ Institut für Theoretische Physik, Universität Regensburg, D-93040 Regensburg \\ ${ }^{b}$ Institut für Physik, Universität Augsburg, D-86135 Augsburg \\ ${ }^{c}$ Department of Physics, University of Cincinnati, Cincinnati, OH 45221
}

\begin{abstract}
Wilson's Numerical Renormalization Group method is used to study the paramagnetic ground state of the periodic Anderson model within the dynamical mean-field approach. For the particle-hole symmetric model, which is a Kondo insulator, we find that the lattice Kondo scale $T_{0}$ is strongly enhanced over the impurity scale $T_{K} ; T_{0} / T_{K} \propto \exp \{1 / 3 I\}$, where $I$ is the Schrieffer-Wolff exchange coupling. In the metallic regime, where the conduction band filling is reduced from one, we find characteristic signatures of Nozières exhaustion scenario, including a strongly reduced lattice Kondo scale, a significant suppression of the states available to screen the f-electron moment, and a Kondo resonance with a strongly enhanced height. However, in contrast to the quantitative predictions of Nozières, we find that the $T_{0} \propto T_{K}$ with a coefficient which depends strongly on conduction band filling.
\end{abstract}

71.27.+a,71.10.Fd,71.20.Eh

\section{INTRODUCTION}

Lanthanide or Actinide-based heavy fermion compounds [1] can be viewed as a paradigm for strong correlation effects in solids. These systems comprise a great variety of different low-temperature states, including paramagnetic metals with either a Fermi liquid or non-Fermi liquid properties [1,2], long range magnetic order, superconductivity or coexistent magnetism and superconductivity [1]. While it is very interesting and challenging to understand the physics of these ordered ground states, not even the basic ingredient, namely the physics of the paramagnetic metal, has been fully captured yet on a microscopic basis. The common understanding is that the physical properties of heavy fermion compounds are largely determined by spin-flip scattering between spins localized on the Lanthanide or Actinide sites and delocalized conduction quasi particles usually formed by the $d$-states in the system. In the case of dilute compounds this spin-flip scattering leads to the well-known Kondo effect, i.e. the screening of the localized spins by the conduction electrons and the formation of a Fermi liquid with a low-energy scale set by the socalled Kondo temperature, $T_{\mathrm{K}}$ depending exponentially and non-perturbatively on the system parameters [3]. Interestingly, the physics of the metallic phase of concentrated systems can in some cases, e.g. $\mathrm{CeAl}_{3}, \mathrm{CeB}_{6}$ or $\mathrm{CeCu}_{6}$ [1], be at least qualitatively understood by a picture of independent, but coherent Kondo scatterers, with the low-energy scale set by the Kondo temperature of the dilute system. However, for $\mathrm{UPt}_{3}, \mathrm{URu}_{2} \mathrm{Si}_{2}$ or $\mathrm{Yb}_{4} \mathrm{As}_{3}$ [4] or the compound $\mathrm{LiV}_{2} \mathrm{O}_{4}$ recently characterized as heavy fermion system [5] there seem to exist two distinct energy scales; one high temperature scale, $T_{\mathrm{K}}$, describing conventional incoherent Kondo scattering, and a much smaller scale, $T_{0}$, also called coherence scale in literature, which marks the onset of Fermi liquid formation characterized by quasi particles with strongly enhanced effective masses.

This apparent discrepancy in the experimental situation has triggered numerous theoretical investigations, from which two dominant competing scenarios have emerged.

One scenario, based on a diagrammatic perturbation theory [6.7], succeeded in approximately mapping the concentrated system onto a set of independent, effective impurities which at low temperature form a coherent Fermi liquid state due to the lattice periodicity. The theory further predicts the existence of a single energy scale which uniquely determines the high energy properties like Kondo screening as well as the formation of the low-temperature Fermi liquid. Most important, apart from moderate renormalizations which usually lead to an enhancement, this lattice scale is given in terms of the energy scale of the corresponding diluted system [7] and the one particle properties close to the Fermi energy are well described by a picture of hybridizing bands, leading to a density of states with a (pseudo-) gap slightly above the Fermi energy [8]. The large effective masses can be accounted for by the observation that the Fermi energy lies in the region with flat bands [7.97. A further theoretical study, based on a variational treatment of the system with a Gutzwiller wave function and employing the Gutzwiller approximation, seems to support this result in the sense that the energy scale calculated is enhanced over the corresponding scale of the dilute system, i.e. there is quite likely only one relevant energy scale. However, this variational scale is more strongly renormalized 10$]$.

The other scenario is based on Noziéres argument that in concentrated systems there will generally not be enough conduction states available to screen all of the localized spins. This situation is engendered by the fact that only the band states within $T_{K}$ of the Fermi surface can effectively participate in screening the local moments. The number of screening electrons can be estimated as $\rho_{0}^{c}(0) T_{K} \ll 1$, where $\rho_{0}^{c}(0)$ is the conduction band density of states at the Fermi level. Thus, in a concentrated system one should encounter an intermediate temperature 
regime where all band states available for screening are "exhausted" - from which the name "exhaustion physics" was coined - and the system starts to resemble an incoherent metal where only part of the spins will be screened [11]. However, since the Kondo screening clouds are not pinned to a particular site they can move through the system with an effective hopping matrix element and a residual strong local correlation, because there can never be two screening clouds on the same site. Based on these arguments Nozières thus suggests, that at low temperatures the system effectively behaves similar to a Hubbard model with a small number of holes and strong local Coulomb repulsion. The remaining entropy can now be quenched by forming a state with either long-range antiferromagnetic order or short ranged antiferromagnetic fluctuations, which give rise to a small low-energy scale and a corresponding heavy Fermi liquid, too [13]. It is important to note that these phenomenological arguments by Nozières do not make any reference to a particular model or specific parameter regime of a model like the periodic Anderson model; thus, if Nozières arguments are correct, exhaustion physics should actually be the generic situation.

Although both scenarios seem to be in accordance with some experimental facts, they apparently fail to capture the whole story. Moreover, in some cases they rely either on approximations that are difficult to control on a microscopic basis or are entirely based on phenomenological arguments, as it is the case with Nozières' point of view. In order to discriminate which ansatz is correct or outline the borders where each of the two scenarios may be valid, a more thorough microscopic study of the properties of the paramagnetic phase is highly desirable.

Recently, Quantum Monte Carlo (QMC) simulations of the periodic Anderson model (PAM) within the Dynamical Mean-Field Theory (DMFT) [12 $\sqrt{14}$ have shown that when the conduction band is nearly half filled [15], there is a single scale consistent with the predictions of Rice and Ueda |10], but obviously inconsistent with Nozières' picture of exhaustion. On the other hand, when the conduction band filling is significantly reduced, the states available for screening of the local moments appear to be depleted near the Fermi surface and the lattice coherence scale is strongly reduced from the corresponding impurity scale 16,17. A protracted evolution of the photoemission spectra 18], and transport [17] are predicted and can be understood in terms of a crossover between the two scales. Although a quantitative relationship between the two scales could not be established, the reduction was ascribed to exhaustion 18]. Nozières subsequently argued that exhaustion should lead to a significant reduction of the lattice scale and predicted that $T_{0}$ is at most $N_{d}(0) T_{K}^{2}$ [19.

In this contribution we present results for the zerotemperature properties of the periodic Anderson model obtained within the DMFT in conjunction with Wilson's numerical renormalization group (NRG) calculations [20]. We will show that for systems with approxi- mately half filled conduction band, one finds a single energy scale, in accordance with the independent impurity picture and which qualitatively behaves as predicted by Rice \& Ueda [10]; moreover, in this parameter regime we do not find any hint towards the occurrence of exhaustion physics; which raises the question to what extent Nozières' phenomenological argument, that only a fraction $\rho_{0}^{c}(0) T_{\mathrm{K}} \ll 1$ of the band states can contribute to the screening of the f-spins, is valid.

In order to observe a behavior at all that is in at least qualitative agreement with Nozières' exhaustion scenario, one actually has to strongly reduce the carrier concentration of the conduction band. For these low-carrier systems the physical behavior completely changes and can now be understood in the framework of exhaustion. However, a quantitative comparison of the lattice energy scale and the one of the corresponding impurity model is possible with our method, and indicates a strikingly different relation between the two than recently predicted by Nozières [19] even in this parameter regime.

The paper is organized as follows. In the next section we will introduce the periodic Anderson model and its treatment within the DMFT using NRG. In section III we will discuss recent developments in understanding the physics of the PAM in some detail and provide a basis to interpret the NRG results presented in section IV. The paper will be concluded by a summary and outlook in section $\mathrm{V}$.

\section{MODEL AND METHOD}

\section{A. Periodic Anderson Model}

The standard model used to describe the physics of heavy fermion systems is the periodic Anderson model (PAM)

$$
\begin{aligned}
H= & \sum_{\mathbf{k} \sigma} \varepsilon_{\mathbf{k}} c_{\mathbf{k} \sigma}^{\dagger} c_{\mathbf{k} \sigma}+\varepsilon_{f} \sum_{\mathbf{k}} f_{\mathbf{k} \sigma}^{\dagger} f_{\mathbf{k} \sigma}+U \sum_{i} n_{i \uparrow}^{f} n_{i \downarrow}^{f} \\
& +\sum_{\mathbf{k} \sigma} V_{\mathbf{k}}\left(c_{\mathbf{k} \sigma}^{\dagger} f_{\mathbf{k} \sigma}+\text { h.c. }\right)
\end{aligned}
$$

In (1), $c_{\mathbf{k} \sigma}^{\dagger}$ creates a conduction quasi particle with momentum $\mathbf{k}$, spin $\sigma$ and dispersion $\varepsilon_{\mathbf{k}}$, with $\frac{1}{N} \sum_{\mathbf{k}} \varepsilon_{\mathbf{k}}=\varepsilon_{c}$ as its center of mass; $f_{\mathbf{k} \sigma}^{\dagger}$ creates an f-electron with momentum $\mathbf{k}$, spin $\sigma$ and energy $\varepsilon_{f}$ and $n_{i \sigma}^{f}$ is the number operator for f-electrons at lattice site $i$ with spin $\sigma$. The localized f-states experience a Coulomb repulsion $U$ when occupied by two electrons and the two subsystems are coupled via a hybridization $V_{\mathbf{k}}$. Although it is in general a crude approximation we will assume for computational reasons $V_{\mathbf{k}}=$ const. for the rest of the paper.

Given the usually rather complex crystal structure of heavy fermion compounds, the question to what extent the simple model (1), which describes orbitally 
non-degenerate f-states, is appropriate. However, especially in Ce-based systems the relevant configuration is $\mathrm{f}^{1}$, whose multiplicity will in general be reduced to a Kramers doublet in the crystal field; the other crystal field multiplets are generally well separated from this ground state doublet [1]. The situation is more complicated in Uranium compounds, where with equal probability a $\mathrm{f}^{2}$ state can be the lowest configuration. In this case the assumption of a Kramers doublet is of course not justified. Nevertheless, even for these compounds the PAM seems to provide an at least qualitatively correct description, so that we assume it to be the relevant model for those compounds, too.

In contrast to e.g. the Hubbard model, where for one dimension an exact solution via Bethe ansatz is available and a combination of many-body techniques was successful to exploit the ground state properties exactly, no such exact benchmark is available for the PAM. The only limit where the physical properties are almost completely known is the impurity version of the model (1) (single impurity Anderson model, SIAM) [3], where a single site with f-states exists which couples to the conduction states. In this limit one finds for $\left|\varepsilon_{f}\right| / V,\left(\varepsilon_{f}+U\right) / V \gg 1$ the mentioned Kondo behavior, namely a screening of the local moment by the conduction states with an energy scale (Kondo temperature)

$$
\begin{aligned}
& T_{\mathrm{K}}=\lambda \sqrt{I} \exp \left(-\frac{1}{I}\right) \\
& I=2 V^{2} \rho_{0}^{c}(0)\left|\frac{1}{\varepsilon_{f}}-\frac{1}{\varepsilon_{f}+U}\right|
\end{aligned}
$$

with $\rho_{0}^{c}(0)$ the density of states at the Fermi energy of the conduction band and $\lambda$ a cut-off energy. Note, that $T_{\mathrm{K}}$ depends exponentially on the system parameters, and especially non-analytically on $V^{2}$.

It is important to stress that the occurrence of the Kondo temperature is intimately connected to the thermodynamic limit, where a (quasi-) continuum of band states exists at the Fermi energy. This property makes reliable calculations with techniques usually suitable to treat finite-sized two or three dimensional systems, like exact diagonalization or quantum Monte Carlo, of limited value, since they can only handle small to moderately sized systems. For the quantum Monte Carlo an additional problem is the sign problem, that becomes increasingly serious as $U$ and the system size increase. Direct perturbational approaches like e.g. FLEX are by construction restricted to small values of $U$ and generally fail to capture even the basics of the Kondo physics. Notable exceptions occur again for the SIAM. Here, straight forward second order perturbation theory in $U$ (not selfconsistent) is able to reproduce at least qualitative features in the special case of full particle-hole symmetry 21,22. More recently, a novel ansatz using a perturbation theory about the unrestricted Hartee-Fock solution for the SIAM 23] has been shown to give even quanti- tatively correct results for spectral functions and energy scales of the SIAM in the Kondo limit 24.

\section{B. Dynamical Mean-Field Theory}

The other non-trivial limit, where an exact solution of the PAM (1) becomes at least in principle possible is the limit of large dimensionality, where the dynamical mean-field theory (DMFT) becomes exact 12 14. Here, the renormalizations due to the local Coulomb repulsion become purely local [12], i.e. one obtains a one-particle self energy independent of momentum

$$
\Sigma^{f}(\mathbf{k}, z) \rightarrow \Sigma^{f}(z)
$$

This property may be used to remap the lattice problem onto an effective SIAM again 25,26]. The non-trivial aspect of the theory comes about by the fact that the medium coupling to the effective impurity is a priori not known but has to be determined self-consistently in the course of the calculation [27. In particular, the selfconsistency relation for the PAM reads

$$
\begin{aligned}
G^{\mathrm{loc}}(z) & =\int d \epsilon \frac{\rho_{0}^{c}(\epsilon)}{z-\varepsilon_{f}-\Sigma^{f}(z)-\frac{V^{2}}{z-\epsilon-\varepsilon_{c}}} \\
& =\frac{1}{z-\varepsilon_{f}-\tilde{\Delta}(z)-\Sigma^{f}(z)} \stackrel{!}{=} G^{\mathrm{SIAM}}(z)
\end{aligned}
$$

with $\rho_{0}^{c}(\epsilon)$ the density of states of the bare conduction band in (11). The second line in (4) defines a generalized hybridization function $\tilde{\Delta}(z)$, which implicitly depends on $\Sigma^{f}(z)$ and is thus modified from its non-interacting form by the presence of correlated f-electrons on other sites in an averaged way.

The remaining task is to solve the effective SIAM defined by the set of parameters $\left\{\varepsilon_{f}, U\right\}$ and the generalized Anderson width

$$
\Gamma(\omega)=-\Im m\{\tilde{\Delta}(\omega+i \eta)\}
$$

Note that the self-consistency condition (4) requires the knowledge of a dynamical quantity, viz the one-particle Green function for all frequencies. This immediately rules out techniques like Bethe ansatz, since it is impossible to calculate dynamical correlation functions with this method. To perform this task nevertheless, a variety of different methods have been developed and applied during the past decade: Quantum Monte Carlo simulations [26], exact diagonalization [14, extended second order perturbation theory (iterated perturbation theory, IPT) [14,29], resolvent perturbation techniques [9,30, localmoment approach [23] and Wilson's NRG 31 33.

In this contribution we used the last method, for the following reasons. First, it is tailored to capture the lowenergy physics of the Kondo problem with high accuracy. Second, it is able to identify exponentially small energy scales. Third, it is non-perturbatively and thus 
also produces the correct dependence of the Kondo temperature on the parameters (see (2)) and, last but not least, it has only little numerical restrictions on the choice of model parameters. Together with past years developments 33, 34 we are able to study the low-temperature properties of the PAM with the NRG with good accuracy.

\section{Details of Wilson's NRG}

Quite generally, the NRG is based on a logarithmic discretization of the energy axis, i.e. one introduces a parameter $\Lambda>1$ and divides the energy axis into intervals $\left[\Lambda^{-(n+1)}, \Lambda^{-n}\right]$ for $n=0,1, \ldots, \infty$ 3,20]. With some further manipulations [33] one can map the original model onto a semi-infinite chain, which can be solved iteratively by starting from the impurity and successively adding chain sites. Since the coupling between two adjacent sites $n$ and $n+1$ vanishes like $\Lambda^{-n / 2}$ for large $n$, the low-energy states of the chain with $n+1$ sites are determined by a comparatively small number $N_{\text {states }}$ of states close to the ground state of the $n$-site system. In practice, one retains only those $N_{\text {states }}$ from the $n$-site chain to set up the Hilbert space for $n+1$ sites and thus prevents the usual exponential growth of the Hilbert space as $n$ increases. Eventually, after $n_{\mathrm{NRG}}$ sites have been included in the calculation, adding another site will not change the spectrum significantly and one terminates the calculation.

It is obvious, that for any $\Lambda>1$ the NRG constitutes an approximation to the system with a continuum of band states but becomes exact in the limit $\Lambda \rightarrow 1$. Performing this limit is, of course, not possible as one has to simultaneously increase the number of retained states to infinity. One can, however, study the $\Lambda$ - and

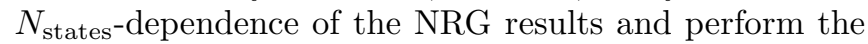
limit $\Lambda \rightarrow 1, N_{\text {states }} \rightarrow \infty$ by extrapolating these data. Surprisingly one finds that the dependence of the NRG results on $\Lambda$ as well as on the cut-off $N_{\text {states }}$ is extremely mild, in most cases is a choice of $\Lambda=2$ and $N_{\text {states }}=300 \ldots 500$ sufficient [24].

While the knowledge of the states is sufficient to calculate thermodynamic properties, the self-consistency (4) requires the knowledge of the one-particle Green function or, equivalently the knowledge of the single-particle self energy $\Sigma^{f}(z)$. Since the NRG scheme works with a discretization of the energy axis, it corresponds to a discrete system and by construction the Green function consists of a set of poles and an appropriate coarse-graining procedure has to be applied. During the last 15 years considerable progress has been made to extract dynamical properties with the NRG, too, and it has been shown to give very accurate results also for e.g. dynamical one- and two-particle and also transport properties 35,36]. For dynamical properties the NRG works best at $T=0$, and various dynamic correlation functions can be calculated with an accuracy of a few percent. Although less well de- fined for finite temperatures, its extension to $T>0$ also shows very good agreement with exact results [32,36]. It is quite remarkable as no sum-rules (Friedel sum rule, total spectral weight etc.) must be used as input for these calculations. On the contrary, they can serve as an independent a posteriori check on the quality of the results. The first application of the NRG to the DMFT known to us is the work of Sakai et al. [31, 32] where the symmetric Hubbard and periodic Anderson model in the metallic regime have been studied. In their papers, the authors point out some difficulties in the progress of iterating the NRG results with the DMFT equations, which are in our opinion largely related to the necessary broadening of the NRG spectra.

As has been shown in the work of Bulla et al. [33], these difficulties can be circumvented if, instead of calculating $G^{f}(z)$ and extracting $\Sigma^{f}(z)$ from it, one calculates the self energy directly via the relation

$$
\begin{aligned}
& \Sigma_{\sigma}^{f}(z)=U \frac{F_{\sigma}^{f}(z)}{G_{\sigma}^{f}(z)} \\
& F_{\sigma}^{f}(z)=\left\langle\left\langle f_{\sigma} f_{\bar{\sigma}}^{\dagger} f_{\bar{\sigma}}, f_{\sigma}^{\dagger}\right\rangle\right\rangle_{z},
\end{aligned}
$$

which originates from the equation of motion

$$
\begin{aligned}
z G_{\sigma}^{f}(z) & =1+\left\langle\left\langle\left[f_{\sigma}, H_{\mathrm{SIAM}}\right], f_{\sigma}^{\dagger}\right\rangle\right\rangle(z) \\
& =1+\left(\varepsilon_{f}+\Delta(z)\right) G_{\sigma}^{f}(z)+U F_{\sigma}^{f}(z)
\end{aligned}
$$

with

$$
\Delta(z)=\frac{1}{N} \sum_{\mathbf{k}} \frac{\left|V_{\mathbf{k}}\right|^{2}}{z-\varepsilon_{\mathbf{k}}} .
$$

Both correlation functions $G_{\sigma}^{f}(z)$ and $F_{\sigma}^{f}(z)$ appearing in (6) can be calculated with the NRG and it turns out that the quotient of them gives a much better result for $\Sigma^{f}(z)$ than the use of $G^{f}(z)$ alone 33 .

Let us note one particular problem in dealing with the PAM in the DMFT+NRG. As we will see in the beginning of section IV], the effective hybridization for the PAM in the particle-hole symmetric limit exhibits a pole right at the Fermi level (see Fig. 3 on page 6). It is clear that such a pole will lead to numerical difficulties. However, the NRG allows to deal with such a structure in a very efficient way, namely by including this single state represented by the pole into the definition of the "impurity" defining the beginning of the NRG chain.

\section{RECENT RESULTS}

Early studies of the PAM using the DMFT concentrated on the particle-hole symmetric case $\varepsilon_{c}=0$, $2 \varepsilon_{f}+U=0$, i.e. $n_{f}=1$ and $n_{c}=1$ 15,32,37. To solve the SIAM, the authors employed QMC 26,28 and NRG [32. Although for this particular situation the system is 
a band insulator for symmetry reasons, one can extract a Wilson Kondo scale from the SIAM impurity susceptibility. Interestingly, this energy scale is enhanced with respect to the corresponding SIAM Kondo temperature in accordance with the results by Rice \& Ueda [10,15]. In addition, at low temperatures the system can order antiferromagnetically 15. To avoid confusion and make the discussion more transparent we will use $T_{0}$ and $T_{\mathrm{K}}$ from now on the distinguish the relevant lattice energy scale and the Kondo scale, respectively.

One possibility to break the particle-hole symmetry is by depleting the band filling via changing $\varepsilon_{c}$, but keeping $n_{f} \approx 1$, which has been done with QMC for $U / V^{2} \approx 4$ 16 18. The resulting phase diagram turns out to be quite interesting for several reasons. First, one finds a suppression of the antiferromagnetic order with decreasing $n_{c}$ and around $n_{c}=0.5$ a region with ferromagnetic order emerges [16], which for $n_{c}<0.5$ can be attributed to a ferromagnetic effective RKKY-type exchange. Second, from studies of the resistivity and optical conductivity [17, 18], one can infer that for $n_{c} \lesssim 0.8$ the physics of the paramagnetic metallic phase drastically change. While for $n_{c} \gtrsim 0.8$ there seem to exist only one energy scale $T_{0} \gtrsim T_{\mathrm{K}}$, the resistivity data suggest, that for $n_{c} \lesssim 0.8$ the onset of coherence is marked by a temperature scale $T_{0} \ll T_{\mathrm{K}}[17,18]$. In addition, the spectral function drastically changes as one decreases $n_{c}$ and the effective Anderson width (5), which for $n_{c} \approx 1$ has a peak at the Fermi energy, starts to develop a dip for $\omega=0$. These observations were taken as fingerprints of Noziéres exhaustion scenario [17, 18]. Further evidence for this interpretation comes from the work by Vidhyadhiraja et al. [38], which is based on $2^{\text {nd }}$ order perturbation theory in $\bar{U}$ (IPT). These authors, too, find a similar behaviour as function of $n_{c}$. In addition, since their method allows to study $T=0$, they could extract the energy scale $T_{0}$ from their data. Interestingly, they found a relation between $T_{0}$ and $T_{\mathrm{K}}$ of the form $T_{0} \propto\left(T_{\mathrm{K}}\right)^{2}$ for their results, which is precisley the behaviour predicted by the phenomenological theory of Noziéres 19]. However, we note that this particular relationship $T_{0} \propto T_{K}^{2}$ in [38] was found only if the width of the Kondo peak was used to estimate $T_{0}$. When the ratio of the masses was used instead, the relationship rather follows $T_{0} \propto T_{K}$ 40.

The estimate of $T_{0}$ coming from Gutzwiller ansatz calculations on the other hand apparently fail to capture the essential physics in this parameter regime, since they predict a ratio $T_{0} / T_{\mathrm{K}}>1$ for all values of $n_{c}$ [1].

Although the results from QMC and IPT are at a first glance very convincing, there remain some problems. First, both series of calculations were done with a comparatively small value of $U / V^{2} \approx 4$ and a systematic study of the behaviour as function of $U / V^{2}$ especially for larger values is clearly necessary. Second, the results were either obtained by QMC [17,18] or IPT [38]. However, for large $U / V^{2}$, the identification of exponentially small energy scales with QMC is problematic due to its restriction to finite temperatures. The IPT on the other hand leads to ambiguous results as mentioned before; in addition, as a perturbational approach in $U$, it certainly cannot produce exponentially small energy scales. Thus, for a quantitative description of the lowtemperature phase and especially a reliable calculation of the low-energy scale $T_{0}$, a non-perturbative technique at $T=0$ is necessary.

\section{NRG RESULTS}

Such a method has become available recently by the application of Wilson's NRG [20] to the DMFT [31] 33], which we use to study the paramagnetic phase of the PAM within the DMFT at $T=0$. In order to perform the energy integral in (4) analytically and to be able to make contact to earlier results we study a simple hypercubic lattice in the limit of dimensionality $d \rightarrow \infty$. With the proper scaling 13 this leads to $\rho_{0}^{c}(\epsilon)=\exp \left(-\left(\epsilon^{2} / t^{*}\right)^{2}\right) /\left(t^{*} \sqrt{\pi}\right)$ for the noninteracting DOS of the band states. In the following we use $t^{*}=1$ as our energy unit.

Let us start by briefly discussing the particle-hole symmetric situation, i.e. $2 \varepsilon_{f}+U=0$ and $\varepsilon_{c}=0$. Here, it is expected from symmetry and shown by calculations [15, 32, 37, that the concentrated system exhibits a hybridization gap. This is shown in Fig. 1, where the $f$ $\operatorname{DOS} A_{f}(\omega)=-\frac{1}{\pi} \Im m\left\{G^{f}(\omega+i \delta)\right\}$ is plotted for the SIAM (dashed line) and the PAM (full line). The model parameters are $U=2$ (i.e. $\varepsilon_{f}=-1$ ) and $V^{2}=0.2$. The result for the SIAM nicely shows the well-known structures, namely the charge excitation peaks at $\omega \approx \pm U / 2$ and the Abrikosov-Suhl resonance (ASR) at the Fermi

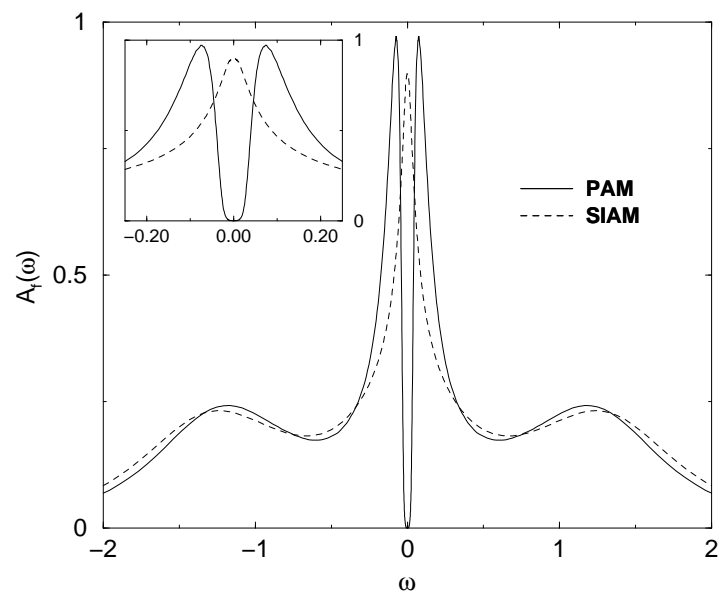

FIG. 1. $f$-density of states $A_{f}(\omega)$ for SIAM (dashed line) and PAM (full line) in the particle-hole symmetric case $2 \varepsilon_{f}+U=0$ and $\varepsilon_{c}=0$. The model parameters are $U=2$ and $V^{2}=0.2$. The inset shows a blowup of the region around the Fermi level.

level. This latter structure, which can be regarded as an effective local level, basically leads to the hybridization 
gap in the PAM. The enlarged view of the region around the Fermi energy also shows, that the width of this hybridization gap and the width of the ASR are of similar order of magnitude. As we will dicuss in a moment the corresponding energy scale in the lattice is actually enhanced over $T_{\mathrm{K}}$, which sets the width of the ASR. This result can readily be anticipated from the self-energy of the $f$-states in Fig. 2. In the SIAM (dashed line) and the PAM (full line) one observes a nice parabolic extremum
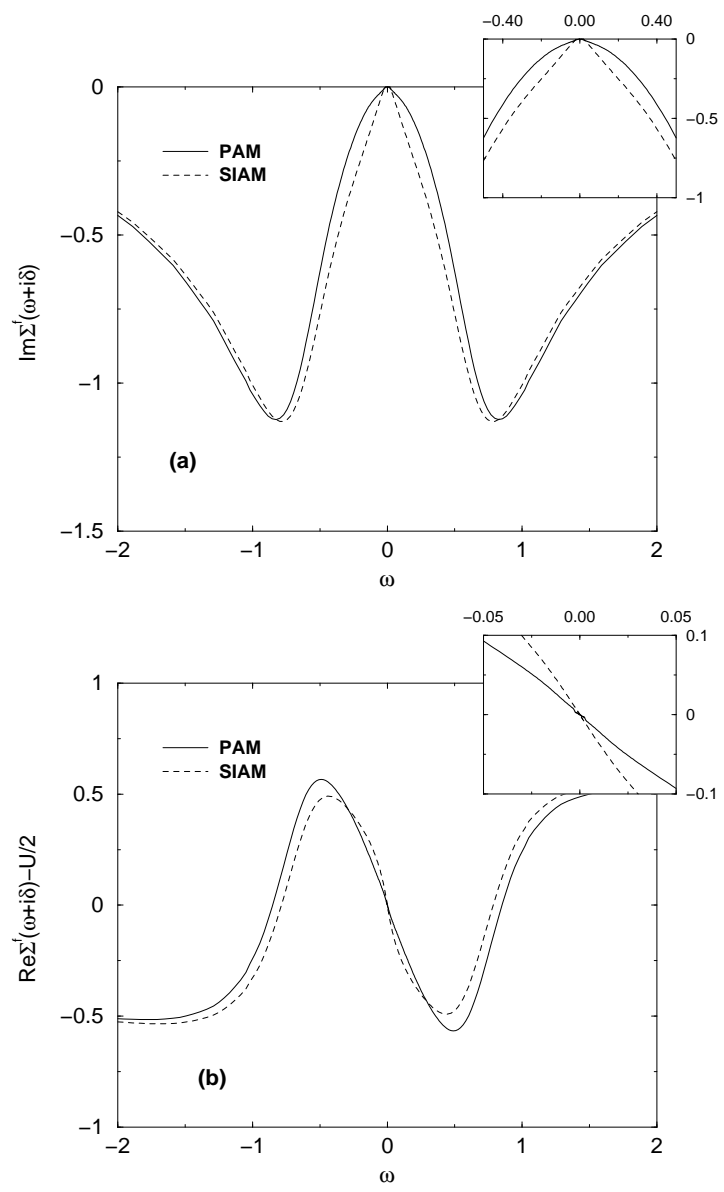

FIG. 2. $\Sigma^{f}(\omega+i \delta)$ SIAM (dashed line) and PAM (full line) in the particle-hole symmetric case and parameters according to Fig. 11. From the real part (b) the Hartree term $U / 2$ has been subtracted. The insets show a blowup of the region around the Fermi level.

in $\Im m \Sigma^{f}(\omega+i \delta)$ at the Fermi energy (see Fig. 2a), which is accompanied by a linear region in $\Re e \Sigma^{f}(\omega+i \delta)$ in Fig. 2b; the slope is negative in both cases and definitely smaller for the PAM, as can be seen from the inset to Fig. $2 \mathrm{~b}$. This means that, at least as far as the self energy is concerned, the system can be viewed as a Fermi liquid with a quasi-particle weight

$$
Z^{-1}=1-\left.\frac{d \Re e \Sigma^{f}(\omega)}{d \omega}\right|_{\omega=0} \equiv m^{*} / m
$$

where we introduced the notion of the effective mass $m^{*}$.
Note that the above result, viz that the system is a Fermi liquid, is not a priori apparent from Fig. 11, since the spectrum represents an insulator. From the self-energy it is however evident that this insulating state can be trivially accounted for by using the picture of hybridizing quasi-particle bands, one of which is located at the Fermi energy and describes an effective f-level. The characterization of the particle-hole symmetric system as band insulator is a priori not the only possible scenario. Indeed, for small $V^{2}$ and $U \rightarrow \infty$ an alternative route to an insulating state is via a Mott-Hubbard transition as found by Held et al. for non-constant $V_{\mathbf{k}}$ [39]. We did not observe such a transition for the case $V_{\mathbf{k}}=$ const. studied here for values of $U$ as large as $U=10$, but also cannot exclude such a scenario on the basis of the data available.

It is also quite illustrating to have a look at the effective Anderson width as defined by (5). This function is rather featureless for the SIAM (dashed line in Fig. 3), but exhibits a very pronounced structure near the Fermi

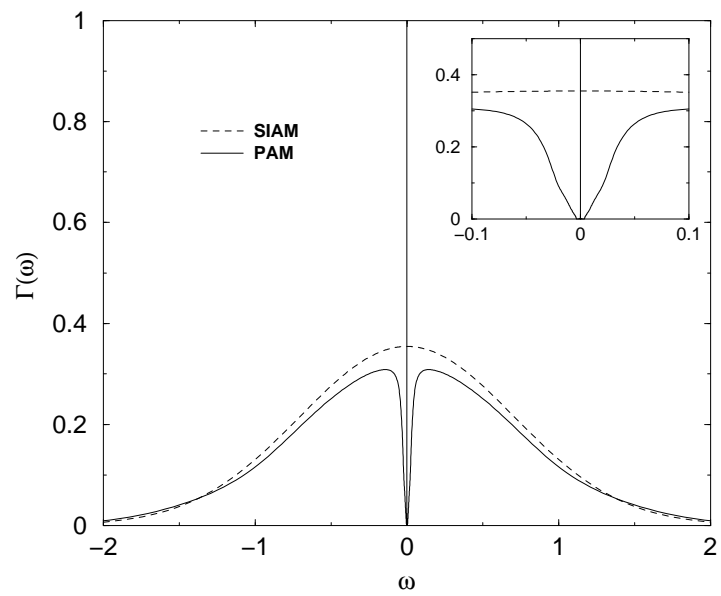

FIG. 3. Anderson width $\Gamma(\omega)$ for SIAM (dashed line) and PAM (full line) in the particle-hole symmetric case and parameters according to Fig. 11. The inset shows a blowup of the region around the Fermi level.

level for the PAM (full line in Fig. 3), namely a pseudo gap plus a strong peak right at $\omega=0$; one can in fact show that the latter is a $\delta$-peak. This $\delta$-peak can be understood as emerging from the sharp quasi-particle band with dominantly f-character at the Fermi level.

The results by QMC and IPT suggest that one can expect drastic changes in the physics of the model if one breaks the particle-hole symmetry. There are actually two possible routes to accomplish this goal; the first is to keep $\varepsilon_{c}=0$, and thus $n_{c} \approx 1$, but increase $U$ so that $2 \varepsilon_{f}+U>0$. An example for the spectrum and the corresponding hybridization function for $\varepsilon_{f}=-1, U=6$ and $V^{2}=0.2$ is shown in Fig. 4 , where we plot the DOS $A_{f}(\omega)$ (Fig. 4a) and the hybridization function $\Gamma(\omega)$ (Fig. $4 \mathrm{~b})$. For these particular parameters the occupancies are $n_{c} \approx 1$ and $n_{f} \approx 0.92$. As in the particle-hole symmetric case one finds the characteristic three-peak structure, 
again with a hybridization gap in the DOS of the lattice

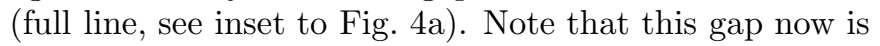
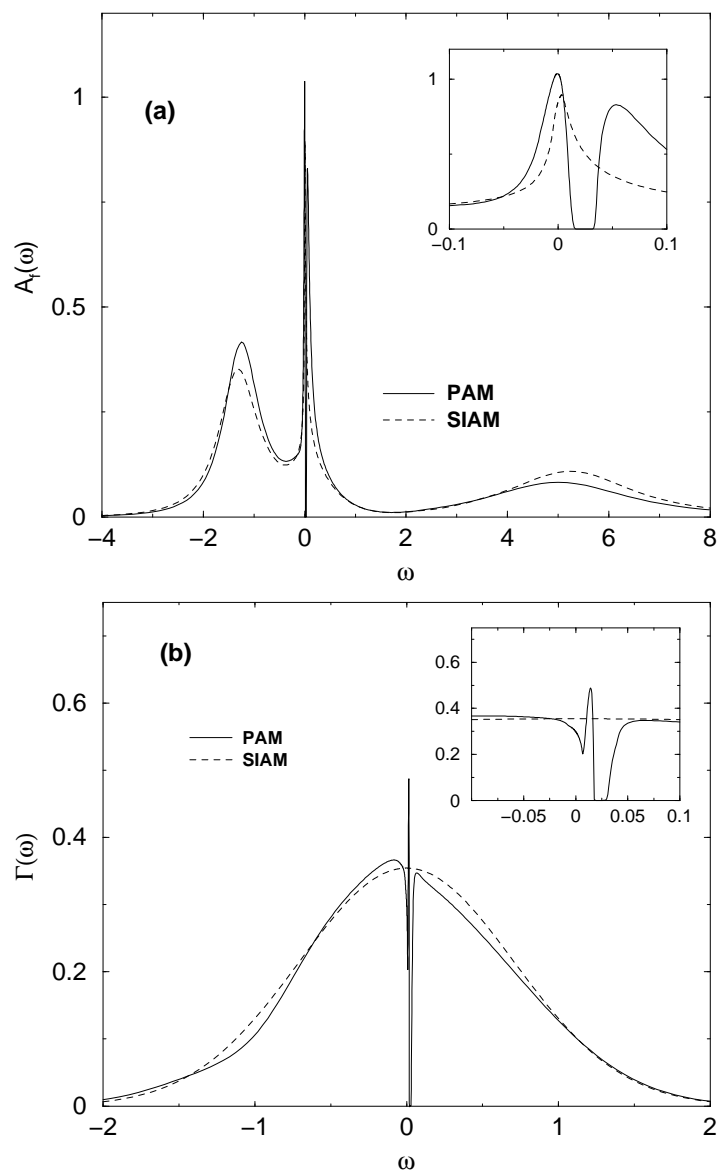

FIG. 4. DOS $A_{f}(\omega)$ (a) and Anderson width $\Gamma(\omega)$ (b) for SIAM (dashed line) and PAM (full line) in the asymmetric case. The parameters are $\varepsilon_{c}=0, \varepsilon_{f}=-1, U=6$ and $V^{2}=0.2$. The insets show a blowup of the region around the Fermi level.

located above the Fermi energy and its width is visibly larger than the width of the ASR in the SIAM; pointing again towards an enhanced energy scale for the lattice, which is also confirmed by an inspection of the self energy. A replica of this (pseudo-) gap is also visible in the effective hybridization function of the lattice in Fig. Фab, which in addition shows a pronounced peak slightly above the Fermi energy. The origin of this peak is the same as for $n_{f}=1$; only it now has to be damped due to the finite lifetime of the quasi-particles for $\omega>0$. It is quite interesting to note that the value of $\Gamma(0)$ is actually reduced from its value in the SIAM, but the average of $\Gamma(\omega)$ over a region of the order $T_{0}$ around the Fermi energy is enhanced. If one assumes that it will be such an averaged value that determines the low-energy scale, one can readily understand that $T_{0}$ is enhanced over $T_{\mathrm{K}}$ by this simple picture. We should mention that our results are very similar to those obtained more than 10 years ago with resolvent perturbation theories [6,7,9].
In fact, the physical situation studied then was basically the same, namely a particle-hole symmetric conduction band hybridizing with an asymmetric $f$-level, however with $U=\infty$. The interpretation of the structures in $\Gamma(\omega)$, which may be viewed as the effective DOS of the medium visible to the impurity, then leads to the picture that due to the coherent admixture of $f$-states to the system, there will be an effective enhancement of $\Gamma(\omega)$ close to the Fermi energy. In this sense one may identify the physics in this region of parameter space with the picture of coherent Kondo scatterers.

The most interesting question is how the energy scales

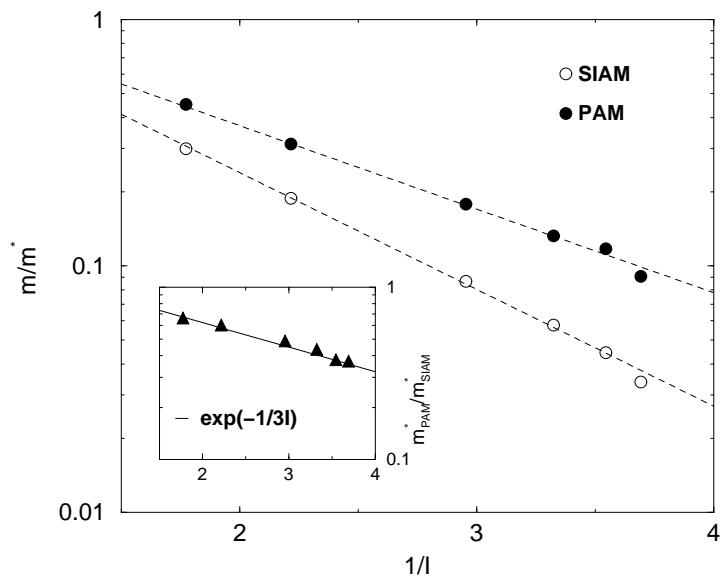

FIG. 5. Inverse effective mass as function of $1 / I$ (see text) for $\varepsilon_{c}=0$ and $\varepsilon_{f}=-1$. The inset shows the ration $m_{\mathrm{PAM}}^{*} / m_{\text {SIAM }}^{*}$ together with a fit $m_{\mathrm{PAM}}^{*} / m_{\text {SIAM }}^{*}=e^{-1 / 3 I}$.

of the dilute system and the lattice, $T_{\mathrm{K}}$ and $T_{0}$, are related in this parameter regime. Let us recall that from the Gutzwiller ansatz one obtains [10] $T_{\mathrm{K}} / T_{0}=$ $m_{\text {PAM }}^{*} / m_{\text {SIAM }}^{*}=\exp (-1 / 2 I)$, where $I=8 \rho_{0}^{c}(0) V^{2} / U$; this prediction was found to be consistent with recent DMFT QMC simulations 15] where the Wilson Kondo scale was estimated form the excess impurity susceptibility.

At $T=0$, the most efficient way to extract the lowenergy scale is by calculating the effective mass according to (8). With $m / m^{*} \propto T_{0}$ we are then able to discuss the variation of $T_{\mathrm{K}}$ and $T_{0}$ with $U$. The result is shown in Fig. 局, where $m / m^{*}$ is plotted versus $1 / I$ and $I=2 \rho_{0}^{c}(0) V^{2} U /\left(\left|\varepsilon_{f}\right|\left(\varepsilon_{f}+U\right)\right)$ is the Schrieffer-Wolff exchange coupling. As already predicted from the spectra and self energies, the general relation $T_{0}>T_{\mathrm{K}}$ holds. In addition, both $T_{\mathrm{K}}$ and $T_{0}$ apparently behave exponentially as function in $1 / I$, but with different slopes; in qualitative agreement with the predictions the slope of $T_{0}$ is smaller. To quantify the relation between $T_{\mathrm{K}}$ and $T_{0}$ further we show in the inset to Fig. 5 the ratio $T_{\mathrm{K}} / T_{0}=m_{\mathrm{PAM}}^{*} / m_{\text {SIAM }}^{*}$ as function of $1 / I$. Again, an exponential behavior is observed; however, in contrast to the predicted factor $1 / 2$ we find $\ln \left(T_{\mathrm{K}} / T_{0}\right)=-1 / 3 I$ from 

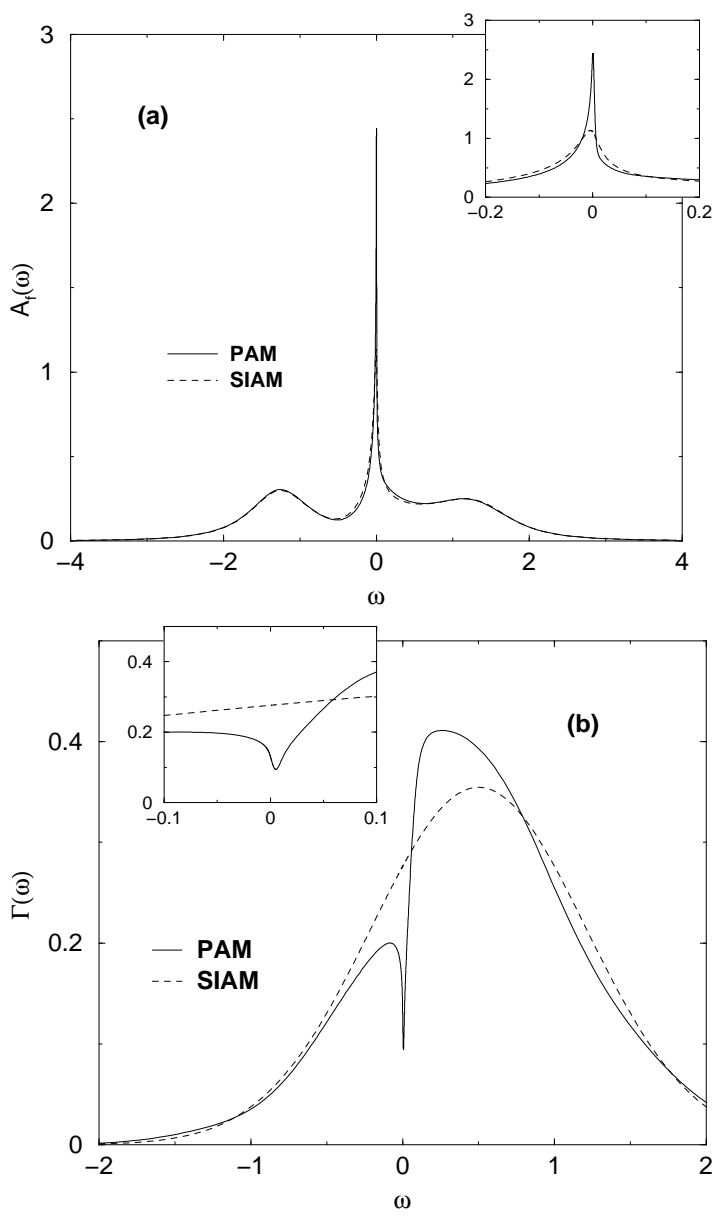

FIG. 6. $f$-density of states $A_{f}(\omega)$ (a) and effective hybridization function $\Gamma(\omega)$ (b) for PAM (dashed line) and SIAM (full line). The parameters are $\varepsilon_{c}=0.5,2 \varepsilon_{f}+U=0$, $U=2$ and $V^{2}=0.2$.

our data. For the time being we do not have an explanation for this discrepancy between the QMC and NRG results; whether it might be related to different schemes to extract $T_{0}$ - scaling behavior of the excess susceptibility as function of temperature in the QMC versus effective mass at $T=0$ in the NRG - has to be clarified.

The second possibility to destroy particle-hole symmetry is to choose $\varepsilon_{c} \neq 0$. As a typical result for that parameter regime we show in Fig. 6 the DOS $A_{f}(\omega)$ (Fig. 6a) and the corresponding effective hybridization function $\Gamma(\omega)$ (Fig. 6 b) for $\varepsilon_{c}=0.5,2 \varepsilon_{f}+U=0, U=2$, and $V^{2}=0.2$. For the filling we obtain $n_{f} \approx 1$ and $n_{c} \approx 0.6$ for both the PAM (dashed line) and the single impurity Anderson model (SIAM, full line). As usual, one sees the characteristic structures, namely the chargeexcitation peaks at $\omega \approx \pm U / 2$ and the Kondo resonance at the Fermi level. However, in contrast to the results with $\varepsilon_{c}=0$, we do not find any hint of a hybridization gap in the lattice DOS; in fact, the DOS looks pretty much like that of a conventional SIAM. The major difference to the DOS of the SIAM is an enhancement of the ASR and a reduction of its width [17, as is apparent from the inset to Fig. 6a. Particularly interesting is the behavior of the effective hybridization function in Fig. 6b. Similar to the case $\varepsilon_{c}=0,2 \varepsilon_{f}+U>0$ it is considerably reduced in the region around the Fermi level; in contrast to the former case, however, the sharp quasiparticle contribution is missing and the average value of $\Gamma(\omega)$ over the region of the order $T_{0}$ around $\omega=0$ is still reduced from the non-interacting value here. The depletion in $\Gamma(\omega)$ around the Fermi level has been coined as hall-mark of exhaustion physics in the PAM and related models [17,38], since according to Nozières phenomenological picture [19] the effective density of medium states available at a given site should be reduced due to screening at other sites.

The fundamental difference in the physics between

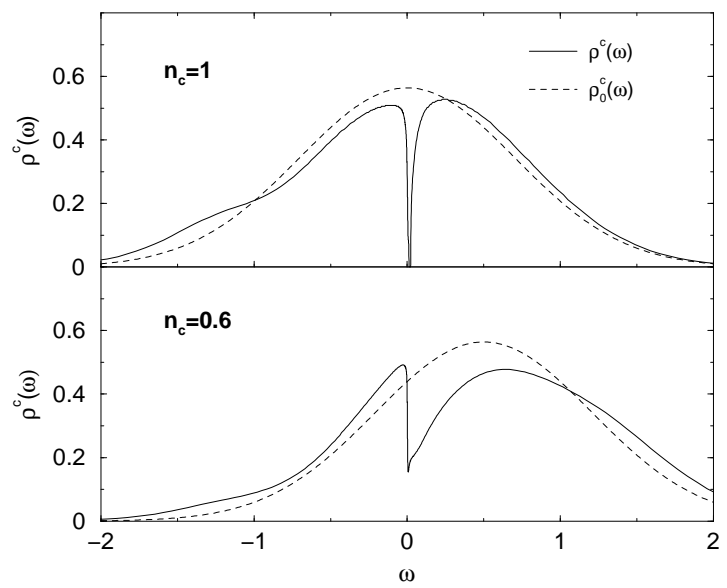

FIG. 7. $c$-density of states $\rho^{c}(\omega)$ of the PAM for $\varepsilon_{f}=-1$, $V^{2}=0.2$ and $U=6, \varepsilon_{c}=0\left(n_{c} \approx 1\right.$, upper panel $)$ and $U=2$, $\varepsilon_{c}=0.5\left(n_{c} \approx 0.6\right.$, lower panel). The dashed lines depict the bare conduction DOS $\rho_{0}^{c}(\omega)$ for the corresponding $\varepsilon_{c}$.

$n_{c} \approx 1$ and $n_{c} \ll 1$ also manifests itself in the behavior of the DOS of the conduction states. Typical results for this quantity are shown in Fig. 7 for $\varepsilon_{f}=-1, V^{2}=0.2$ and $\varepsilon_{c}=0, U=6\left(n_{c} \approx 1\right.$, upper panel) and $\varepsilon_{c}=0.5$, $U=2\left(n_{c} \approx 0.6\right.$, lower panel). For comparison, the bare band DOS for the corresponding value of $\varepsilon_{c}$ is also included (dashed curves); the choice of different values of $U$ for the cases $\varepsilon_{c}=0$ and $\varepsilon_{c}=0.5$ is necessary to ensure that both systems are metallic. One observes fundamental qualitative differences in the DOS, especially close to the Fermi surface, which in our opinion are related to the different physical properties of the different regimes and do not depend critically on a particular choice of the interaction strength. For $n_{c} \approx 1$, the appearance of a gap slightly above the Fermi level in the conduction DOS again supports the notion of hybridizing bands in this region of parameter space. On the other hand, the conduction DOS for the case $n_{c} \approx 0.6$ does not show the typical form of hybridized bands, but merely a pseudogap at the Fermi energy. The fact, that the spectrum only develops a pseudo-gap with finite DOS for $\omega=0$, 
can again be understood as sign of exhaustion, since the formation of a full hybridization gap requires complete Kondo screening by the band states to occur for each f-site; while the formation of a pseudo-gap can be interpreted that only part of the f-sites are screened by the conduction electrons.

The reduced effective hybridization around the Fermi level observed in Fig. 6 gives also rise to a reduced lowenergy scale, characterized by an effective mass $m^{*} / m \approx$ 17 in the PAM, whereas the corresponding quantity for the SIAM is $m^{*} / m \approx 8$. The behavior of the low-energy scale as function of $n_{c}$ for fixed $U=2, V^{2}=0.25$ and

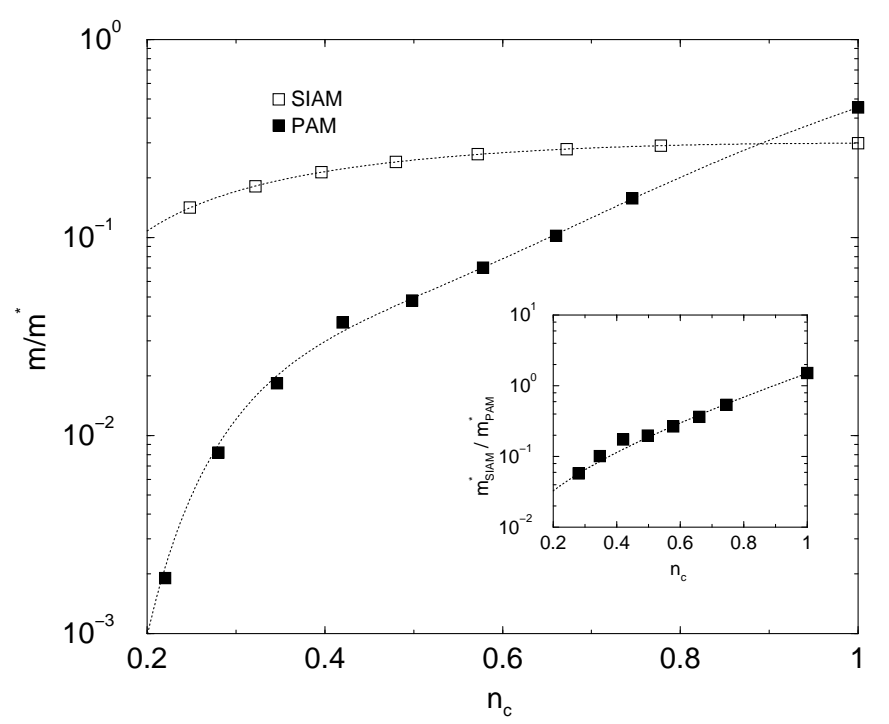

FIG. 8. $m / m^{*}$ vs. $n_{c}$ for $U=2, V^{2}=0.25$ and $n_{f} \approx 1$. The lines are guides to the eye. The inset shows the ratio $m_{\mathrm{SIAM}}^{*} / m_{\mathrm{PAM}}^{*} \equiv T_{0} / T_{\mathrm{K}}$ versus $n_{c}$. The data are fit by $T_{0} / T_{\mathrm{K}} \propto n_{c} \exp \left(c \cdot n_{c}\right)$ (full line), where $c \approx 5 / 2$.

$n_{f} \approx 1$ is shown in Fig. 8. Note that for $n_{c} \approx 1$ the value $T_{0}$ is again enhanced over the impurity scale with $\ln \left(T_{\mathrm{K}} / T_{0}\right)=-1 / 3 I$. Below $n_{c} \approx 0.8$ the ratio $T_{0} / T_{K}$ decreases below one and falls rapidly and monotonically with $n_{c}$, being almost two orders of magnitude smaller for $n_{c} \approx 0.2$. The ratio $m_{\mathrm{SIAM}}^{*} / m_{\mathrm{PAM}}^{*} \equiv T_{0} / T_{\mathrm{K}}$ is shown in the inset to Fig. 8. This ratio falls more rapidly than $T_{\mathrm{K}}$, i.e. $T_{0} / T_{\mathrm{K}}^{2}$ is not constant. The ratio $T_{0} / T_{\mathrm{K}}$ can be fit to a form $T_{0} / T_{\mathrm{K}} \propto n_{c} \cdot \exp \left(c \cdot n_{c}\right)$ with $c \approx 5 / 2$ (Full curve in the inset to Fig. 8). It gives an excellent account of the data. This behaviour especially means that $T_{0} \sim n_{c} T_{\mathrm{K}}$ as $n_{c} \rightarrow 041$. Note that the Gutzwiller result for $n_{c} \ll 1$ predicts both $T_{0}$ and $T_{\mathrm{K}}$ to behave like $n_{c} e^{-c \cdot n_{c}}$, but (i) predicts $T_{0} / T_{\mathrm{K}}>1$ and (ii) clearly gives no proportionality to $n_{c}$ in the ratio $T_{0} / T_{\mathrm{K}}$.

Nozières phenomenological arguments also lead to an estimate of $T_{0}$ as function of $n_{c}$, namely $T_{0} \propto$ $\left(T_{K}\right)^{2} / \rho_{0}^{c}(0)[19$. This relation has recently been tested with IPT 38 and, within this approach, found to be fulfilled at least for $U / V^{2} \approx 4$ between $0.4 \leq n_{c} \leq 0.8$ when $T_{0}$ is estimated from the width of the Kondo resonance. We first note, that clearly our result in Fig. 8 is not compatible with this prediction. In order to clarify the relation between $T_{0}$ and $T_{\mathrm{K}}$ we compare $T_{0}$ and $T_{K}$

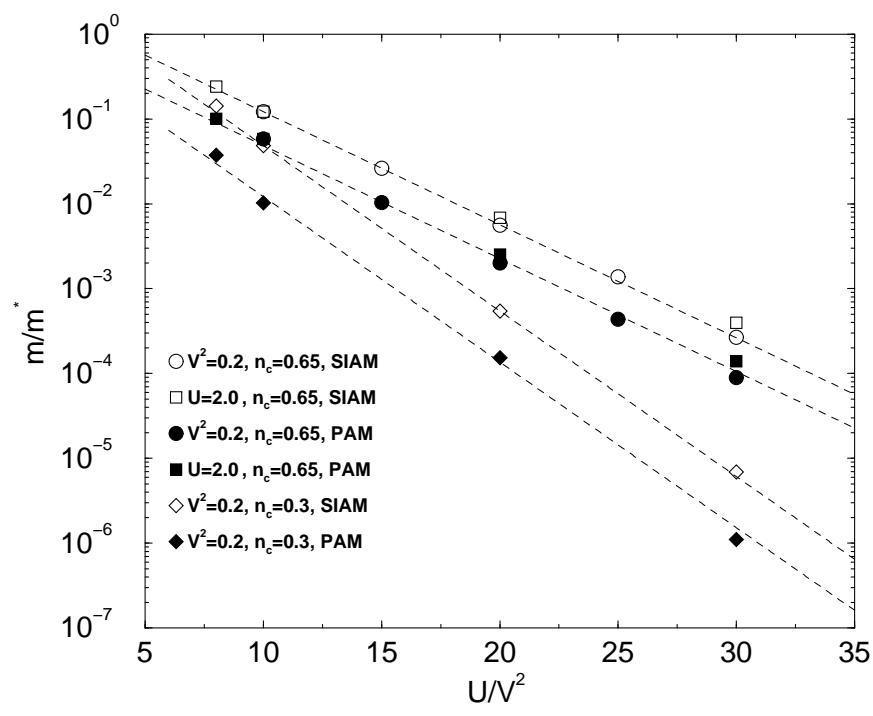

FIG. 9. $\mathrm{m} / \mathrm{m}^{*}$ as function of $U / V^{2}$ for $n_{c} \approx 0.6$ (squares and circles) and $n_{c} \approx 0.31$ (diamonds) with $n_{f} \approx 1$. The circles (squares) denote varying $U\left(V^{2}\right)$ for fixed $V^{2}=0.2$ $(U=2)$. The diamonds show the behaviour of $m / m^{*}$ for a different filling $n_{c} \approx 0.3$ and $V^{2}=0.2$ for varying $U$.

as function of $U / V^{2}$ for fixed $n_{c}$. The results for $n_{c} \approx 0.6$ as function of $U / V^{2}$ for both varying $U$ at constant $V^{2}=0.2$ (circles) and varying $V^{2}$ at constant $U=2$ (squares) is shown in Fig. 9 on a semi-logarithmic scale; to study the dependence on the filling $n_{c}$, we included calculations for $n_{c}=0.31$ (diamonds, varying $U$ only). Evidently $T_{K}$ and $T_{0}$ both follow an exponential law $T_{0}, \quad T_{K} \propto \exp (-a / I)$, where $I=8 \rho_{0}^{c}(0) V^{2} / U$ is the Schrieffer-Wollf exchange coupling for $2 \varepsilon_{f}+U=0$. However, the curves for the SIAM and PAM for fixed $n_{c}$ are parallel in the semi-logarithmic plot in Fig. 9, i.e. the coefficients of $U / V^{2}$ in the exponents of both quantities are identical. This of course means $T_{0} \propto T_{K}$ rather than $T_{0} \propto\left(T_{K}\right)^{2}$, as predicted by Nozières. Thus, neither as function of $n_{c}$ nor as function of $U / V^{2}$ does the lattice scale $T_{0}$ obey Nozières prediction.

\section{SUMMARY AND CONCLUSION}

We have presented results for the PAM obtained within the DMFT at $T=0$ using Wilson's NRG approach. For the range of parameters studied here, the system can always be characterized as a Fermi liquid with a strongly enhanced effective mass; this lattice scale $T_{0}$ is enhanced over a corresponding impurity Kondo scale $T_{\mathrm{K}}$ for the particle-hole symmetric conduction band in accordance with perturbational results [6,7, 9] or those from 
Gutzwiller ansatz [10]. Moreover, the picture of hybridizing quasi particle bands leading to (pseudo-) gaps in the DOS was found to be valid here. On the other hand, in the case of an asymmetric conduction band and especially for low carrier concentration $n_{c} \lesssim 0.8$, the spectral functions and corresponding effective hybridization functions show the signs of exhaustion and we observe a corrresponding strong reduction of the lattice scale $T_{0}$ [16, 19.

Together with our results, an extensive picture of exhaustion physics in the infinite-dimensional PAM has begun to emerge. Close to half filling the low-temperature properties of the model can be characterized by one energy scale $T_{0}>T_{K}$; whereas away from half filling, two scales are apparent: $T_{K}$, where screening begins, and $T_{0} \ll T_{K}$ where coherence sets in. At low temperatures $T \approx T_{0}$, the quasi particle features in the single-particle spectra become pronounced and have predominantly $f$ character [18]. Since only the states near the Fermi energy can participate in screening, the f-electron moments are screened predominantly by other f-electron states. At a temperature $T_{m}\left(T_{0}<T_{m}<T_{K}\right)$, a dip begins to develop in the effective hybridization rate at the Fermi surface $\Gamma(\omega \approx 0)$ 17], indicating that the states available for screening are becoming exhausted. This is a direct confirmation of the qualitative features of Nozières exhaustion scenario. Nevertheless, for fixed $n_{c}$, we find that $T_{0} \propto T_{K}$, and as the conduction band filling changes we find $T_{0} \propto n_{c} \exp \left(c n_{c}\right)$, both are in direct contradiction with the predictions of Nozières. Thus, we conclude that Nozières phenomenological arguments are too crude to capture the quantitative features of exhaustion.

To our knowledge there has not yet been a systematic experimental study concentrating on the signatures of exhaustion in metallic HF compounds. However, there are several experimentally relevant consequences of exhaustion predicted by DMF calculations. Most of these predictions are related to the presence of two relevant scales and the protracted behavior of measurements as a function of temperature in crossover regime between these scales [16]. For example, as compared to the predictions of the SIAM with the same $T_{0}$, the photoemission peak should evolve much more slowly with temperature [18]. In addition, it should have significantly more weight since the height of the peak goes like $1 / \Gamma(0)$ and its width is set by $T_{0}$. Although these features have been reported in photoemission experiments on Yb-based HF materials 42, these results remain controversial 43] and it has been suggested that the experimental spectrum is representative of the surface, and not the bulk 44]. Fortunately, transport and neutron scattering experiments probe much further into the bulk, and should display characteristic features due to exhaustion. The calculated resistivity displays a non-universal peak and two other regions typical of HF systems and associated with impurity-like physics at high temperatures $T \approx T_{K}$ and Fermi liquid formation at low $T \lesssim T_{0}[17]$. The peak resistivity shows features characteristic of exhaustion. It occurs at $T \approx T_{m}$, the temperature at which exhaustion first becomes apparent as a dip $\Gamma(\omega \approx 0)$. $T_{m}$ is nonuniversal in that it increases with decreasing $n_{c}, T_{K}$ and $T_{0}$. The Drude peak in the optical conductivity persists up to much higher temperatures than predicted by the SIAM and the Drude weight weight rises dramatically with temperature [17]. The quasielastic peak in the angle integrated dynamic spin susceptiblity also evolves more slowly with temperature than predicted by the SIAM, and it displays more pronounced charge-transfer features [46].

Despite the rich picture which has begun to emerge from DMF calculations, many questions remain unresolved. Among these, three seem most prominent to us. First, it is unclear what is happening as $n_{c} \rightarrow 1$. In this regime, following Nozières argument, there should also remain too few states to screen the moments so the exhaustion scenerio would seem to apply, too; nevertheless, the $T_{0}$ is enhanced relative to $T_{K}$ and all effects of exhaustion vanish. It is tempting to attribute this vanishing of exhaustion to another energy scale associated with the bare gap that appears in the spectra as $n_{c} \rightarrow 1$. However, in recent calculations for a model with f-d hopping such that the hybridization $V_{k} \propto \epsilon_{k}$, where there is no conduction band gap when $n_{f}=n_{c}=1$, in the regime of strong f-d hybridization $T_{0} \gg T_{K}$ is again found, suggesting that there must be some more fundamental reason for the absence of exhaustion 45. Thus, these latter results together with the ones presented here, surely demand for a critical reinvestigation of the phenomenology of exhaustion.

Second, thus far, all calculations are for the orbitally non-degenerate models. The effect of orbital degeneracy and crystal field splittings has yet to be determined. However, in the limit of infinite orbital degeneracy, the Kondo scale would seem to be unrenormalized.

Third, following Doniach's arguments, RKKY interaction and superexchange will compete with Kondo screening in the formation of the ground state. In the present work, we have explicitely concentrated on the paramagnetic state, i.e. these types of exchange do not enter the calculation. However, within the DMF it is possible to study the influence of RKKY or superexchange on the mean-field level by either looking at the susceptibility [15] or allowing for a symmetry-broken state. Generally, since the RKKY exchange grows like $J^{2}$, and the Kondo scale is exponential in $-1 / J$, Kondo screening and hence exhaustion is expected to be most pronounced when the Kondo exchange $J \sim V^{2} / U$ is large. However, we have found that exhaustion can dramatically reduce the relevant low-energy scale; which may extend the region where the magnetic exchange dominates in the formation of the ground state. Thus a systematic study of the magnetic phase diagram as function of $U / V^{2}$ is clearly desirable.

Acknowledgements: It is a pleasure to acknowledge fruitful discussions with D. Logan, H.R. Krishanmurthy, F. Anders, A. Georges, N. Grewe, J. Keller, D. Vollhardt. 
One of us (MJ) would like to acknowledge support by the NSF via grants DMR9357199 and DMR 9704021. This work was in part supported by a NATO collaborative research travel grant.

[1] For reviews see N. Grewe and F. Steglich, Handbook on the Physics and Chemistry of Rare Earths, edited by K.A. Gschneidner, Jr. and L.L. Eyring (Elsevier, Amsterdam, 1991), Vol. 14, p. 343; D.W. Hess, P.S. Riseborough and J.L. Smith, Enc. of Applied Physics edited by G. L. Trigg (VCH Publishers Inc., New York, 1991), Vol. 7, p. 435.

[2] See e.g. Conference on Non-Fermi liquid Physics, J. Cond. Matter 8, 9675(1997).

[3] A. C. Hewson, The Kondo Problem to Heavy Fermions, Cambridge Univ. Press (1993).

[4] J. Schoenes, C. Schönenberger, J.J.M. Franse and A.A. Menovski, Phys. Rev. B35, 5375(1987); S. Kimura, A. Ochiai, T. Suzuki, Physica B 230-232, 705(1997).

[5] S. Kondo, D.C. Johnston, C.A. Swenson, F. Borsa, A.V. Mahajan, L.L. Miller, T. Gu, A.I. Goldman, M.B. Maple, D.A. Gajewski, E.J. Freeman, N.R. Dilley, R.P. Dickey, J. Merrin, K. Kojima, G.M. Luke, Y.J. Uemura, O. Chmaissem, and J.D. Jorgensen, Phys. Rev. Lett. 78, 3729(1997); S. Kondo, D.C. Johnston and L.L. Miller, Phys. Rev. B59, 2609(1999).

[6] C. Kim, Y. Kuramoto and T. Kasuya, J. Phys. Soc. Jpn. 59, 2414(1990).

[7] N. Grewe, T. Pruschke and H. Keiter, Z. Phys. B 71, $75(1988)$.

[8] D.L. Cox and N. Grewe, Z. Phys. B71, 321(1988).

[9] Th. Pruschke and N. Grewe, Z. Phys. B74,439(1989).

[10] T.M. Rice and K. Ueda, Phys. Rev. B34, 6420(1986).

[11] P. Nozières, Ann. Phys. Fr. 10, 19-35(1985).

[12] W. Metzner and D. Vollhardt, Phys. Rev. Lett. 62, 324 (1989); see also E. Müller-Hartmann, Z. Phys. B 74, 507 (1989).

[13] Th. Pruschke, M. Jarrell and J.K. Freericks, Adv. in Phys. 42, 187 (1995).

[14] A. Georges, G. Kotliar, W. Krauth and M.J. Rozenberg: Rev. Mod. Phys. 68, 13(1996).

[15] M Jarrell, Phys. Rev. B51, 7429 (1995).

[16] A.N. Tahvildar-Zadeh, M. Jarrell and J.K. Freericks, Phys. Rev. B55, R3332(1997).

[17] A.N. Tahvildar-Zadeh, M. Jarrell, Th. Pruschke and J.K. Freericks, Phys. Rev. B60, 10782(1999).

[18] A.N. Tahvildar-Zadeh, M. Jarrell and J.K. Freericks, Phys. Rev. Lett. 80, 5168(1998).

[19] P. Nozières, Eur. Phys. B 6, 447-457(1998).

[20] K.G. Wilson, Rev. Mod. Phys. 47, 773(1975); H.R. Krishna-murthy, J.W. Wilkins and K.G. Wilson, Phys. Rev. B21, 1003 \& 1044(1980).

[21] K. Yosida and H. Kojima, Prog. Theor. Phys. 46, 244(1970).

[22] V. Zlatiź and B. Horvatić, Phys. Rev. B28, 6904(1983).
[23] D. Logan, M.P. Eastwood and M.A. Tusch, J. Phys.: Condens. Matter 10, 2673(1998).

[24] R. Bulla, M. Glossop, D. Logan and Th. Pruschke, to appear in Journal of Physics.

[25] C. Mielsch and U. Brandt, Z. Phys. B75, 365(1989); V. Janiś, Z. Phys. B83 227(1991); A. Georges and G. Kotliar, Phys. Rev. B45, 6479 (1992).

[26] M. Jarrell, Phys. Rev. Lett. 69, 168(1992).

[27] For a detailed review of the DMFT and the selfconsistency cycle we refer the reader to 13,14.

[28] J.E. Hirsch and R.M. Fye, Phys. Rev. Lett. 56, 2521 (1989); M. Jarrell and J.E. Gubernatis, Physics Reports Vol. 269 No.3, p133-195, (May, 1996).

[29] A. Martín-Rodero, F. Flores, M. Baldo and R. Pucci, Solid State Commun. 44, 991(1982); A. Levy Yeyati, A. Martín-Rodero and F. Flores, Phsy. Rev. Lett. 71, 2991(1993); H. Kajueter and G. Kotliar, Phys. Rev. Lett. 77, 131(1996).

[30] H. Keiter, J. C. Kimbal, Phys. Rev. Lett. 25, (1970) 672; N. E. Bickers, D. L. Cox, J. W. Wilkins, Phys. Rev. B36, (1987) 2036.

[31] O. Sakai and Y. Kuramoto, Sol. Stat. Comm. 89 307(1994).

[32] Y. Shimizu and O. Sakai, in Computational Physics as a New Frontier in Condensed Matter Research" (eds. H. Takayama et al., The Physical Society of Japan, 1995), p.42.

[33] R. Bulla, Th. Pruschke and A.C. Hewson J. Phys.: Cond. Matter 9 10463(1997); J. Phys.: Cond. Matter 10, 8365(1998); R. Bulla, Phys. Rev. Lett. 83, 126(1999).

[34] Th. Pruschke, R. Bulla and M. Jarrell, to appear in Physica B.

[35] O. Sakai, Y. Shimizu and T. Kasuya, J. Phys. Soc. Jpn. 58, 3666(1989).

[36] T.A. Costi, A. Hewson and V. Zlatić, J. Phys.: Cond. Matter 6, 2519(1994).

[37] M. Jarrell, H. Akhlaghpour and Th. Pruschke, Phys. Rev. Lett. 70, 1670(1993).

[38] N.S. Vidhyadhiraja, A.N. Tahvildar-Zadeh, M. Jarrell and H. R. Krishnamurthy, cond-mat/9905408.

[39] K. Held, C. Huscroft, R. Scalettar and A.K. McMahan, cond-mat/9905011.

[40] We thank A. Georges for suggesting this calculation.

[41] M. Grilli, G. Kotliar and A.J. Millis, Phys. Rev. B42, 329(1990).

[42] R.I.R. Blyth, J.J. Joyce, A.J. Arko, P.C. Canfield, A.B. Andrews, Z. Fisk, J.D. Thompson, R.J. Bartlett, P. Riseborough, J. Tang and J.M. Lawrence, Phys. Rev. B48, 9497 (1993).

[43] For example see L.H. Tjeng, S.-J. Oh, E.-J. Cho, H.J. Lin, C. T. Chen, G.-H. Gweon, J.-H. Park, J.W. Allen, T. Suzuki, M.S. Makivic and D.L. Cox, Phys. Rev. Lett. 711419 (1993); J.J. Joyce, A.J. Arko, A.B. Andrews and R.I.R. Blyth, Phys. Rev. Lett. 72, 1774 (1994); L.H. Tjeng, S.-J. Oh, C.T. Chen, J.W. Allen and D.L. Cox, Phys. Rev. Lett. 72, 1775 (1994).

[44] F. Reinert, R. Claessen, G. Nicolay, D. Ehm. S. Hüfner, W.P. Ellis, G.-H. Gweon, J.W. Allen, B. Kindler and W. Assmus, Phys. Rev. B58, 12808 (1998).

[45] K. Majumdar, et al., preprint. 
[46] C. Huscroft, Th. Pruschke, M. Jarrell, in preparation. 